\title{
A NOTE ON A TYPE OF APPROXIMATE IDENTITY IN THE FOURIER ALGEBRA
}

\author{
BRIAN FORREST AND MAHATHEVA SKANTHARAJAH
}

(Communicated by J. Marshall Ash)

\begin{abstract}
Let $A(G)$ denote the Fourier algebra of the locally compact group $G$. We show that for a large class of groups an ideal $I$ in $A(G)$ has a $\Delta$-weak bounded approximate identity if and only if it has a bounded approximate identity.
\end{abstract}

Let $\mathscr{A}$ denote a commutative Banach algebra with structure space $\Delta(\mathscr{A})$. A net $\left\{u_{\alpha}\right\}_{\alpha \in \mathscr{U}}$ in $\mathscr{A}$ is called a $\Delta$-weak bounded approximate identity if there exists $M<\infty$ such that $\left\|u_{\alpha}\right\|_{\mathscr{A}} \leq M$ for every $\alpha \in \mathscr{U}$ and if

$$
\lim _{\alpha}\left|\varphi\left(u_{\alpha} u\right)-\varphi(u)\right|=0 \text { for every } u \in \mathscr{A}, \varphi \in \Delta(\mathscr{A}) .
$$

$\Delta$-weak approximate identities were introduced in [5], where they were called weak bounded approximate identities, a term that is often used for another class of bounded approximate identities. The authors showed that it is possible for an algebra $\mathscr{A}$ to have a $\Delta$-weak bounded approximate identity but not a bounded approximate identity. In this note, we show that this is not the case for ideals in the Fourier algebra of a large class of locally compact groups.

Let $G$ be a locally compact group. Let $A(G)$ denote the Fourier algebra of $G$ as defined by Eymard in [1]. $A(G)$ is a commutative Banach algebra of continuous functions on $G$ with structure space $G$. Given a closed subset $A$ of $G$, we define the ideal $I(A)$ as follows:

$$
I(A)=\{u \in A(G) \mid u(x)=0 \text { for every } x \in A\} .
$$

Given an ideal $I$ of $A(G)$, we define

$$
Z(I)=\{x \in G \mid u(x)=0 \text { for every } u \in I\} .
$$

We say that $A \in \mathscr{R}_{c}(G)$ if $A$ is closed in $G$ and $A \in \mathscr{R}\left(G_{d}\right)$, the ring of subsets of $G$ generated by all left cosets of subgroups of $G_{d}$, where $G_{d}$ denotes $G$ with the discrete topology.

Theorem 1. Let I be a closed ideal in $A(G)$. If I has a $\Delta$-weak bounded approximate identity, then $Z(I) \in \mathscr{R}_{c}(G)$.

Proof. Let $\left\{u_{\alpha}\right\}_{\alpha \in \mathscr{U}}$ be a $\Delta$-weak bounded approximate identity in $I$. Let $A=Z(I)$. If $X \in G \backslash A$, then there exists $u \in I$ with $\operatorname{supp} u \cap A=\varnothing$ and

Received by the editors July 9, 1991 and, in revised form, May 11, 1992.

1991 Mathematics Subject Classification. Primary 43A07, 43A15; Secondary 46J10.

Key words and phrases. Fourier algebra, bounded approximate identity, ideals. 
$u(x)=1$. Since $\lim _{\alpha} u_{\alpha} u(x)=u(x)=1$, it follows that $\left\{u_{\alpha}\right\}_{\alpha \in \mathscr{U}}$ converges pointwise to $1_{G \backslash A}$. However, $u_{\alpha} \in B\left(G_{d}\right)$, the Fourier-Stieltjes algebra of $G_{d}$, and $\left\{u_{\alpha}\right\}_{\alpha \in \mathscr{U}}$ is bounded in $B\left(G_{d}\right)$. It follows from [1, Corollary 2, p. 202] that $1_{G \backslash A} \in B\left(G_{d}\right)$ and, hence, that $1_{A} \in B\left(G_{d}\right)$. The result now follows from Host's noncommutative version of Cohen's idempotent theorem [4].

The next result follows immediately from Theorem 1 and [2, Theorem 3.11]. Corollary 2. Let $G$ be an amenable [SIN]-group. Let I be a closed ideal in $A(G)$. Then the following are equivalent:

(i) I has a $\Delta$-weak bounded approximate identity.

(ii) I has a bounded approximate identity.

(iii) $Z(I) \in \mathscr{R}_{C}(G)$.

We answer a question raised in [4, p. 157].

Corollary 3. Let $G$ be a locally compact abelian group. Let I be a closed ideal in $L^{1}(G)$. Then I has a $\Delta$-weak bounded approximate identity if and only if $Z(I) \in \mathscr{R}_{C}(G)$.

Finally, we remark that for discrete groups the existence of a $\Delta$-weak bounded approximate identity in $A(G)$ can easily be shown to imply the amenability of $G$. Consequently, by verifying that the proof of [2, Theorem 3.20] holds for $\Delta$-weak bounded approximate identities, we see that for discrete $G$ an ideal $I$ of $A(G)$ has a $\Delta$-weak bounded approximate identity if and only if it has a bounded approximate identity. Such ideals can be completely characterized in terms of $Z(I)$ (see [2, Theorem 3.20]).

\section{REFERENCES}

1. P. Eymard, L'algèbre de Fourier d'un groupe localement compact, Bull. Soc. Math. France 92 (1964), 181-236.

2. B. Forrest, Amenability and ideals in $A(G)$, J. Austral. Math. Soc. Ser. A 53 (1992), 143-155.

3. O. Hatori and S. Takahasi, Commutative Banach algebras which satisfy a BochnerSchoenberg-Eberlein type theorem, Proc. Amer. Math. Soc. 110 (1990), 149-158.

4. B. Host, Le thèoréme des idempotents dans $B(G)$, Bull. Soc. Math. France 114 (1986), 215-233.

5. C. Jones and C. Lahr, Weak and norm approximate identities are different, Pacific J. Math. 72 (1977), 99-104.

Department of Pure Mathematics, University of Waterloo, Waterloo, Ontario, CANADA N2L 3G1 